\title{
A FAMILY OF FUNCTIONS AND ITS THEORY OF CONTACT 1
}

\author{
J. F. RITT
}

Introduction. If $p_{1}, \cdots, p_{n}$ are fixed positive integers and $a_{1}, \cdots, a_{n}$ arbitrary constants, it is possible so to choose the $a_{i}$ as to make the function

$$
y(x)=\prod_{i=1}^{n}\left(x-a_{i}\right)^{p_{i}}
$$

and its first $p_{1}+\cdots+p_{n}-1$ derivatives equal to zero for any single value $x_{0}$ of $x$. This is accomplished by taking each $a_{i}$ equal to $x_{0}$. One might say, on this basis, that the family of polynomials (1) has contact of order $p_{1}+\cdots+p_{n}-1$, for every value of $x$, with $y=0$.

A more interesting situation is met when we allow the $p_{i}$ to be any fixed positive numbers, not necessarily integral. In that case $y(x)$ may be a function of many branches, with the quotient of any two branches equal to a constant of modulus unity. For our purposes it suffices to consider the value zero of $x$. If no $a_{i}$ is zero, each branch of $y(x)$ will be analytic at $x=0$, with an expansion

$$
c_{0}+c_{1} x+\cdots+c_{s} x^{s}+\cdots
$$

where the $c_{j}$ depend on the $a_{i}$. The question which we examine is: What is the greatest value of $s$ such that, by suitably varying the $a_{i}$, the coefficients $c_{0}, \cdots, c_{s}$ can be made to approach zero simultaneously? Such a greatest value of $s$ exists, and will be called, below, the order of contact of the family (1) with $y=0$. Denoting the greatest value of $s$ by $r$, we shall prove that

$$
r \leqq q+n-1
$$

where $q$ is the greatest integer less than $p_{1}+\cdots+p_{n}$. When no proper subset of the $p_{i}$ has an integral sum, the equality sign holds in (2). For $n=2,(2)$ can be an inequality only when $p_{1}$ and $p_{2}$ are both integers. For $n \geqq 3,(2)$ will certainly be an inequality if some integral power of $y(x)$ is a polynomial of degree not exceeding $q+n-1$; thus the order of contact of the family

Received by the editors April 9, 1942.

1 The problem of this note was suggested by the considerations of our paper On the singular solutions of algebraic differential equations, Ann. of Math. (2) vol. 37 (1936) p. 552. See also, W. C. Strodt, Trans. Amer. Math. Soc. vol. 45 (1939) p. 276. 


$$
y(x)=\left(x-a_{1}\right)^{1 / 2}\left(x-a_{2}\right)^{1 / 2}\left(x-a_{3}\right)^{1 / 2}
$$

is two rather than three. Whether this describes all exceptional cases for $n \geqq 3$ is not decided here.

1. The family of functions. In what follows, the $p_{i}$ in (1) will be any fixed positive numbers. A few words are necessary to make clear the meaning of the second member of (1) for given $a_{i}$. If the $a_{i}$ are distinct from one another, we may take any simply connected area containing no $a_{i}$ and form the product, in the area, of any selection of branches of the $n$ functions $\left(x-a_{i}\right)^{p_{i}}$. The various products obtainable in this way are continuations of one another and are all branches of a single analytic function, which we consider the second member of (1) to represent. If two or more $a_{i}$ coincide, two distinct products, as just described, need not be branches of the same analytic function. There may thus be more than one, possibly even a countable infinitude of interpretations of the second member of (1); every such analytic function will be accepted into the $n$-parameter family of functions (1).

Given any function $y$, as in (1), its values, for any $x$ which is not an $a_{i}$, are equal in modulus; the same is true for every derivative of $y$.

2. Order of contact. Let $\mathcal{F}$ be a family of analytic functions and $f(x)$ a function ${ }^{2}$ analytic at a point $x_{0}$. There may exist non-negative integers $s$ which have the property that, for every $\epsilon>0$, a $g(x)$ exists in $\mathcal{F}$, with a branch analytic at $x_{0}$, such that, for this branch of $g(x)$, $g(x)-f(x)$ and its first $s$ derivatives are less than $\epsilon$ in modulus at $x_{0}$. If such integers $s$ exist, and if the set of them is bounded, we shall represent the greatest of them by $r$ and shall say that $\mathcal{F}$ has contact of order $r$ with $f(x)$ at $x_{0}$. If the $s$ are unbounded, we shall say that $\mathcal{F}$ has contact of infinite order with $f(x)$ at $x_{0}$.

3. The bound. We examine now the functions (1). It is apparent that this family has contact of some order with $y=0$ at every point. Indeed, because the family is invariant under the addition of any constant to $x$, the contact with $y=0$ is the same for all values of $x$.

Let $q$ be the greatest integer less than $p_{1}+\cdots+p_{n}$. The order of contact of the family (1) with $y=0$ is not less than $q$. This is seen by taking all $a_{i}$ equal to zero. We prove the theorem:

THEOREM. The order of contact which the family (1) has with $y=0$, for every $x$, does not exceed $q+n-1$.

\footnotetext{
${ }^{2}$ Not necessarily in $\mathcal{F}$.
} 
The theorem is readily seen to be true for $n=1$; we employ induction with respect to $n$. We examine the theorem for $n=r>1$, assuming that it has been established for every $n$ less than $r$.

We suppose the theorem false for $n=r$. Then, for $n=r$, and for certain positive numbers $p_{1}, \cdots, p_{r}$ which stay fixed during our proof, the family (1) has contact with $y=0$, for $x=0$, of order greater than $q+r-1$. Thus, if we denote the $j$ th derivative of $y$ by $y_{j},{ }^{3}$ we can, for every $\epsilon>0$, fix the $a_{i}$ in (1) at values distinct from 0 so as to have ${ }^{4}$

$$
\left|y_{i}(0)\right|<\epsilon, \quad i=0,1, \cdots, q+r .
$$

Let us show that, if $\epsilon$ is sufficiently small, each $a_{i}$, as just fixed, will have a modulus less than unity. Suppose, for instance, that for some very small $\epsilon,\left|a_{1}\right| \geqq 1$. Then $y(x) /\left(x-a_{1}\right)^{p_{1}}$ will be very small, together with its first $q+r$ derivatives, at $x=0$. This, by the case of $n=r-1$, is impossible.

We now put

We have

$$
\begin{aligned}
& \alpha(x)=\left(x-a_{1}\right) \cdots\left(x-a_{r}\right) \\
& \beta(x)=\alpha(x)\left[\frac{p_{1}}{x-a_{1}}+\cdots+\frac{p_{r}}{x-a_{r}}\right] .
\end{aligned}
$$

$$
\alpha(x) y_{1}-\beta(x) y=0 .
$$

The polynomial $\beta$ is of degree $r-1$. Its $(r-1)$ st derivative is

$$
(r-1) !\left(p_{1}+\cdots+p_{r}\right) \text {. }
$$

We differentiate (5) $j-1$ times, where $j \geqq 1$. Indicating derivatives of $\alpha$ and $\beta$ by subscripts, we find that

$$
\begin{gathered}
\alpha y_{j}+\left[(j-1) \alpha_{1}-\beta\right] y_{j-1}+\left[\frac{(j-1)(j-2)}{2 !} \alpha_{2}-(j-1) \beta_{1}\right] y_{j-2} \\
+\cdots-\beta_{j-1} y=0 .
\end{gathered}
$$

For $j \geqq r,(7)$ becomes, because of the degrees of $\alpha$ and $\beta$,

(8) $\alpha y_{j}+\cdots+(j-1) !\left[\frac{\alpha_{r}}{r !(j-r-1) !}-\frac{\beta_{r-1}}{(r-1) !(j-r) !}\right] y_{j-r}=0$.

${ }^{3} y_{0}=y$.

4 If $y$ is analytic at $x=0$ when certain $h$ of the $a_{i}$, say $a_{1}, \cdots, a_{h}$, are zero while no other $a_{i}$ vanish, it must be that $p_{1}+\cdots+p_{h}$ is integral. Thus, if $a_{1}, \cdots, a_{h}$ are changed to a common value slightly different from zero, $y$ and any specified finite set of its derivatives will undergo only a slight change at $x=0$. 
The coefficient of $y_{j-r}$ in (8) is a constant, which, if we have regard to (6) and notice that $\alpha_{r}=r$ !, is seen to be zero if and only if

$$
p_{1}+\cdots+p_{r}=j-r .
$$

Let $p$ represent $p_{1}+\cdots+p_{r}$. If, in (1), the $a_{i}$ are all multiplied by a number $m$, the values of $y_{j}(0)$ are multiplied by $m^{p-j}$. If $|m|>1$, each $y_{j}(0)$ with $j>q$ will be multiplied by a number of modulus not greater than unity.

We consider a $y(x)$, (with definite $a_{i}$ ), which satisfies (3) for some very small $\epsilon$. Let $m$ be such that the greatest of the quantities $\left|m a_{i}\right|, i=1, \cdots, r$, has unity for modulus. Then, by what follows (3), $|m|>1$. Let

$$
\bar{y}(x)=\prod_{i=1}^{r}\left(x-m a_{i}\right)^{p_{i}} .
$$

We inspect the relation (8) as formed for $\bar{y}$. First we let $j=q+r$. In that case, (9) cannot hold. Every $\left|\bar{y}_{i}(0)\right|$ with $q<i \leqq q+r$ is small. Furthermore, because $\left|m a_{i}\right| \leqq 1, i=1, \cdots, r$, there are bounds, independent of $\epsilon$, for the values of the coefficients in (8) at $x=0$. We infer that $\left|\bar{y}_{q}(0)\right|$ is small. Now, supposing that $q>0$, let $j=q+r-1$. We find from (8) that $\left|\bar{y}_{q-1}(0)\right|$ is small. Continuing, we find that every $\left|\bar{y}_{i}(0)\right|$ with $i \leqq q+r$ is small.

Let $g$ be such that $\left|m a_{g}\right|=1$. Then the function

$$
\bar{y}(x) /\left(x-m a_{o}\right)^{p}
$$

is small, together with its first $q+r$ derivatives, for $x=0$. It is clear that we can use a single $g$ and obtain a sequence of functions (10) which is such that the values at $x=0$ of the $k$ th function of the sequence and its first $q+r$ derivatives tend toward zero as $k$ increases. By the case of $n=r-1$, this is impossible. The theorem is proved.

4. Attainment of bound. We prove, for $n>1$, the theorem :

TheOREM. If no proper subset of the $p_{i}$ has an integral sum, the family (1) has, for every $x$, contact with $y=0$ of order precisely $q+n-1$.

It suffices to show that, when the $p_{i}$ satisfy the hypothesis, there are values of the $a_{i}$ distinct from zero such that $y_{j}(0)=0$, $j=q+1, \cdots, q+n-1$. Such $a_{i}$ being found, we can multiply them by a small $m$ distinct from zero and obtain a function (1) which is small, for $x=0$, together with its first $q+n-1$ derivatives.

The existence of $a_{i}$ as just described will be established if we can prove that there are numbers $b_{1}, \cdots, b_{n}$, distinct from zero, such that the function 


$$
z=\prod_{i=1}^{n}\left(1+b_{i} x\right)^{p_{i}}
$$

has derivatives, from the $(q+1)$ st to the $(q+n-1)$ st inclusive which vanish for $x=0$. The $n-1$ derivatives in question, which we represent by $Z_{q+1}, \cdots, Z_{q+n-1}$, are homogeneous polynomials in the $n$ letters $b_{i}$. When the $Z_{q}$ are equated to zero, they determine a nonvacuous algebraic manifold each of whose essential irreducible components is of dimension not less than unity. ${ }^{5}$ Thus there is at least one set of numbers $b_{1}, \cdots, b_{n}$ which annul the $Z_{j}$ and are not all zero. We assume in what follows that there is such a set in which the $b_{i}$ are not all distinct from zero, and prove that some proper subset of the $p_{i}$ has an integral sum.

We may now work under the assumption that, for some integer $t$ with $0<t<n$, there exist numbers $c_{1}, \cdots, c_{t}$, all distinct from zero, such that the function

$$
u=\prod_{i=1}^{t}\left(1+c_{i} x\right)^{p_{i}}
$$

has derivatives from the $(q+1)$ st to the $(q+n-1)$ st inclusive which vanish for $x=0$. If we put $d_{i}=-1 / c_{i}$, we find that the function

$$
v=\prod_{i=1}^{t}\left(x-d_{i}\right)^{p_{i}}
$$

has derivatives from order $q+1$ through order $q+n-1$ which vanish for $x=0$. For the derivatives $v_{j}$ of $v$, there exists a relation, analogous to (7), which expresses each $v_{j}$ in terms of the derivatives which precede it if $j \leqq t$, and in terms of the $t$ derivatives which precede it if $j>t$. In this relation, the coefficient of $v_{j}$ is $(-1)^{t} d_{1} \cdots d_{t}$ when $x=0$. Thus, as $v_{q+1}, \cdots, v_{q+n-1}$ vanish for $x=0$, and as they include the $t$ derivatives which precede $v_{q+n}, v_{q+n}$ and, then, all the derivatives which follow it, vanish for $x=0$. In other words, $v$ is a polynomial. Thus $p_{1}+\cdots+p_{t}$ is integral and the theorem is proved.

When the $p_{i}$ are not all integers, $Z_{q+1}$ consists of at least two terms. It is then possible to annul $Z_{q+1}$ with $b_{i}$ which are all distinct from zero, so that, by what precedes, the order of contact is at least $q+1$. In particular, when $n=2$, the order of contact is $q+1$ except when $p_{1}$ and $p_{2}$ are both integers.

\section{Columbia University}

\footnotetext{
${ }^{5}$ van der Waerden, Einfiihrung in die algebraische Geometrie, Berlin, 1939, $\$ 41$.
} 